REVIEW ARTICLE

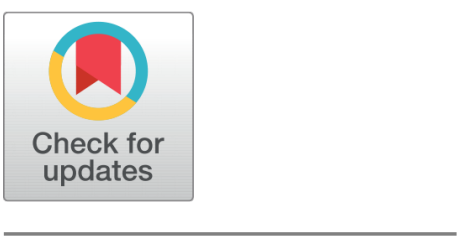

OPEN ACCESS

Received: 08-04-2020

Accepted: 23-04-2020

Published: 25-05-2020

Editor: Dr. Natarajan Gajendran

Citation: Singh Chauhan I, Chauhan TS (2020) D-homothetic deformation of an $\eta$-Einstein Para-Sasakian manifold. Indian Journal of Science and Technology 13(13): 1435-1439. https ://doi.org/10.17485/IJST/v13i13.198

* Corresponding author. Indiwar Singh Chauhan

Assistant Professor, Department of Mathematics, Bareilly College,

Bareilly (U.P.), India

indiwarsingh.chauhan@gmail.com

Funding: None

Competing Interests: None

Copyright: (c) 2020 Singh Chauhan, Chauhan. This is an open access article distributed under the terms of the Creative Commons

Attribution License, which permits unrestricted use, distribution, and reproduction in any medium, provided the original author and source are credited.

Published By Indian Society for Education and Environment (iSee)

\section{D-homothetic deformation of an $\eta$-Einstein Para-Sasakian manifold}

\author{
Indiwar Singh Chauhan ${ }^{1 *}$, T S Chauhan ${ }^{2}$ \\ 1 Assistant Professor, Department of Mathematics, Bareilly College, Bareilly (U.P.), India \\ 2 Associate Professor, Department of Mathematics, Bareilly College, Bareilly (U.P), India
}

\section{Abstract}

Objectives: The main purpose of this paper is to study the theory of Dhomothetic deformation of an $\eta$-Einstein Para-Sasakian manifold. Methods: A deformation technique is employed to solve the resulting problem. We provide its application in the general theory of relativity. Findings: Section 2 deals with recurrent and symmetric Para-Sasakian Manifolds. In section 3 we have defined and studied projectively symmetric Para-Sasakian manifold. The notion of $\phi$-holomorphic sectional curvature in an $\eta$-Einstein Para-Sasakian manifold has been delineated in the section 4 . In this very section, we shall study the relevant commutation formulae which give rise to the required curvature tensor. The obtained results were compared with the previous works ${ }^{(1--3)}$ in this field and were found to be in good agreement. Novelty/Conclusion: The study concludes the following results:

1. If n-dimensional Para-Sasakian manifold is to be recurrent projective symmetric then the recurrent projective curvature tensor vanishes identically.

2. If $\mathrm{n}$-dimensional Para-Sasakian manifold is to be projective semi-symmetric then the semi-recurrent projective curvature tensor vanishes identically.

3. If $\mathrm{n}$-dimensional Para-Sasakian manifold is to be projective scalar-symmetric then the scalar projective curvature vanishes identically.

4. If $\mathrm{D}$-homothetic deformation of $\eta$-Einstein Para-Sasakian manifold is constant $\phi$-holomorphic sectional curvature then we shall obtain the value of scalar fields.

Keywords: $\phi$-holomorphic sectional curvature; constant curvature; Para-Sasakian manifold; recurrent projective symmetric; D-homothetic deformation; $\eta$-Einstein manifold

\section{Introduction}

We consider an $\mathrm{n}$-dimensional differentiable manifold $\mathrm{M}^{\mathrm{n}}$ with a positive definite metric $\mathrm{g}_{\alpha \beta}$ which admits a unit covariant vector field $\eta_{\alpha}$ satisfying
(1.1) $\nabla_{\alpha} \eta_{\beta}-\nabla_{\beta} \eta_{\alpha}=0$
and
(1.2) $\nabla_{\alpha} \nabla_{\beta} \eta_{\gamma}=-\left(g_{\alpha \beta} \eta_{\gamma}-g_{\alpha \gamma} \eta_{\beta}\right)+2 \eta_{\alpha} \eta_{\beta} \eta_{\gamma}$ 
wherein $\nabla_{\alpha}$ denotes the operator of covariant differentiation with regard to $\mathrm{g}_{\alpha} \beta$, such a space $\mathrm{M}^{\mathrm{n}}$ is called Para-Sasakian manifold ${ }^{(4,5)}$.

It is easy to verify that in a Para-Sasakian manifold, the following relations holds good ${ }^{(4,5)}$ :

(1.3) $\eta_{\alpha}=g_{\alpha \beta} \xi^{\beta}$,

(1.4) $\eta_{\alpha} \xi^{\alpha}=1$,

(1.5) $g^{\alpha \beta} \eta_{\beta}=\xi^{\alpha}$,

(1.6) $g^{\alpha \beta} \eta_{\alpha} \eta_{\beta}=1$,

(1.7) $g_{\alpha \beta} \xi^{\alpha} \xi^{\beta}=1$,

(1.8) $\phi_{\alpha \beta}=\nabla_{\alpha} \eta_{\beta}$,

(1.9) $\phi_{\beta}^{\alpha}=\nabla_{\beta} \xi^{\alpha}$,

(1.10) $\phi_{\beta}^{\alpha} \xi^{\beta}=0$,

(1.11) $\phi_{\beta}^{\alpha} \eta_{\alpha}=0$,

(1.12) $\phi_{\beta}^{\alpha} \phi_{\gamma}^{\beta}=-\delta_{\gamma}^{\alpha}+\eta_{\gamma} \xi^{\alpha}$,

(1.13) $g_{\alpha \beta} \phi_{\gamma}^{\alpha} \phi_{\theta}^{\beta}=-g_{\gamma \theta}+\eta_{\gamma} \eta_{\theta}$

(1.14) $\eta_{\lambda} R_{\alpha \beta \gamma}^{\lambda}=g_{\alpha \gamma} \eta_{\beta}-g_{\beta \gamma} \eta_{\alpha}$

and

(1.15) $\eta_{\lambda} R_{\alpha}^{\lambda}=-(n-1) \eta_{\alpha}$

\section{Recurrent and Symmetric Para-Sasakian Manifolds}

\section{Definition 2.1:}

If the Para-Sasakian manifold satisfies the condition

(2.1) $\nabla_{\rho} R_{\alpha \beta \gamma}^{\varepsilon}=\tau_{\rho} R_{\alpha \beta \gamma}^{\varepsilon}$

is called recurrent Para-Sasakian manifold for some non-zero recurrence vector $\tau_{\rho}$ and $R_{\alpha \beta \gamma}^{\varepsilon}$ is a recurrent curvature tensor ${ }^{(5)}$.

If the covariant derivative of recurrent curvature tensor $R_{\alpha \beta \gamma}^{\varepsilon}$ vanishes everywhere then an n-dimensional Para-Sasakian manifold is called recurrent symmetric Para-Sasakian manifold.

As a consequence of this definition follows the result:

(2.2) $\nabla_{\rho} R_{\alpha \beta \gamma}^{\varepsilon}=0$.

Definition 2.2:

If the Para-Sasakian manifold satisfies the condition

(2.3) $\nabla_{\rho} R_{\alpha \beta}=\tau_{\rho} R_{\alpha \beta}$

then the manifold is called Ricci-recurrent Para-Sasakian manifold or semi-recurrent Para-Sasakian manifold for some nonzero recurrence vector $\tau_{\rho}$ and $R_{\alpha \beta}$ is a Ricci-recurrent curvature tensor.

If the covariant derivative of Ricci-recrrent curvature tensor $R_{\alpha \beta}$ vanishes everywhere then an $\mathrm{n}$-dimensional Para-Sasakian manifold is called Ricci-symmetric Para-Sasakian manifold or semi-symmetric Para-Sasakian manifold.

As a consequence of this definition follows the result:

(2.4) $\nabla_{\rho} R_{\alpha \beta}=0$.

Definition 2.3:

If the Para-Sasakian manifold satisfies the condition

(2.5) $\nabla_{\rho} R=\tau_{\rho} R$

then the manifold is called scalar-recurrent Para-Sasakian manifold for some non- zero recurrence vector $\tau_{\rho}$.

If the covariant derivative of scalar curvature $\mathrm{R}$ vanishes everywhere then an $\mathrm{n}$-dimensional Para-Sasakian manifold is called scalar-symmetric Para-Sasakian manifold.

As a consequence of this definition follows the result:

(2.6) $\nabla_{\rho} R=0$.

In this regard, we have the following theorems:

Theorem 2.1:

The necessary and sufficient condition for the $\mathrm{n}$-dimensional Para-Sasakian manifold to be recurrent symmetric manifold is that the recurrent curvature tensor vanishes.

Proof:

Since the recurrent curvature tensor vanishes i.e. $R_{\alpha \beta \gamma}^{\varepsilon}=0$, 
Inserting above in equation (2.1), we obtain $\nabla_{\rho} R_{\alpha \beta \gamma}^{\varepsilon}=0$,

This manifests that the Para-Sasakian manifold to be recurrent symmetric space.

Conversely, If the space to be recurrent symmetric then in view of the above discussion converse of the theorem is proof from the equations (2.1) and (2.2).

Theorem 2.2:

The necessary and sufficient condition for the n-dimensional Para-Sasakian manifold to be Ricci-symmetric manifold is that the Ricci-recurrent curvature tensor vanishes.

Proof:

The proof is self evident in view of the equations (2.3) and (2.4).

Theorem 2.3:

The necessary and sufficient condition for the n-dimensional Para-Sasakian manifold to be scalar symmetric manifold is that the scalar curvature vanishes.

Proof:

It is evident in view of the equations (2.5) and (2.6).

\section{Projectively Symmetric Para-Sasakian Manifold}

\section{Definition 3.1:}

If the Para-Sasakian manifold satisfies the condition

(3.1) $\nabla_{\rho} P_{\alpha \beta \gamma}^{\varepsilon}=\tau_{\rho} P_{\alpha \beta \gamma}^{\varepsilon}$

then the manifold is called Para-Sasakian manifold with recurrent projective curvature tensor for some non-zero recurrence vector $\tau_{\rho}$.

If the covariant derivative of recurrent projective curvature tensor $P_{\alpha \beta \gamma}^{\varepsilon}$ vanishes everywhere then an n-dimensional ParaSasakian manifold is called recurrent projective symmetric Para-Sasakian manifold.

As a consequence of this definition follows the result ${ }^{(6)}$ :

(3.2) $\nabla_{\rho} P_{\alpha \beta \gamma}^{\varepsilon}=0$.

Definition 3 .2:

If the Para-Sasakian manifold satisfies the condition

(3.3) $\nabla_{\rho} P_{\alpha \beta}=\tau_{\rho} P_{\alpha \beta}$

then the space is called Para-Sasakian manifold with semi-recurrent projective curvature tensor for some non-zero recurrence vector $\tau_{\rho}$.

If the covariant derivative of semi-recurrent projective curvature tensor $P_{\alpha \beta}$ vanishes everywhere then an n-dimensional Para-Sasakian manifold is called projective semi-symmetric Para-Sasakian manifold.

As a consequence of this definition follows the result:

(3.4) $\nabla_{\rho} P_{\alpha \beta}=0$.

Definition 3.3:

If the Para-Sasakian manifold satisfies the condition

(3.5) $\nabla_{\rho} P=\tau_{\rho} P$

then the space is called Para-Sasakian manifold with scalar-recurrent projective curvature for some non-zero recurrence vector $\tau_{\rho}$.

If the covariant derivative of scalar projective curvature $\mathrm{P}$ vanishes everywhere then an $\mathrm{n}$-dimensional Para-Sasakian manifold is called projective scalar-symmetric Para-Sasakian manifold.

As a consequence of this definition follows the result:

(3.6) $\nabla_{\rho} P=0$.

In this regard, we have the following theorems:

Theorem 3.1:

The necessary and sufficient condition for the $n$-dimensional Para-Sasakian manifold to be recurrent projective symmetric manifold is that the recurrent projective curvature tensor vanishes.

Proof:

Since the recurrent projective curvature tensor vanishes i.e. $P_{\alpha \beta \gamma}^{\varepsilon}=0$,

Inserting above in equation (3.1), we obtain $\nabla_{\rho} P_{\alpha \beta \gamma}^{\varepsilon}=0$.

This manifests that the Para-Sasakian manifold to be recurrent projective symmetric space. 
Conversely, If the space to be recurrent projective symmetric then in view of above discussion, converse of the theorem is immediately proof from the equations (3.1) and (3.2).

Theorem 3.2:

The necessary and sufficient condition for the $\mathrm{n}$-dimensional Para-Sasakian manifold to be projective semi-symmetric manifold is that the semi-recurrent projective curvature tensor vanishes.

Proof:

The proof is self-evident in view of equations (3.3) and (3.4).

Theorem 3.3:

The necessary and sufficient conditions for the n-dimensional Para-Sasakian manifold to be projective scalar-symmetric manifold is that the scalar projective curvature vanishes.

Proof:

It is evident in view of the equations (3.5) and (3.6).

\section{4 -Holomorphic Sectional Curvature:}

\section{Definition 4.1:}

Para-Sasakian manifold is termed as $\eta$-Einstein manifold whose Ricci tensor $\mathrm{R}_{-}\{\backslash$ alpha $\backslash$ beta $\}$ satisfies the condition ${ }^{(2)}$ :

(4.1) $R_{\alpha \beta}=\tau_{1} g_{\alpha \beta}+\tau_{2} \eta_{\alpha} \eta_{\beta}$,

Wherein $\tau_{1}$ and $\tau_{2}$ are some scalars.

In this regard, we have the following theorem:

\section{Theorem 4.1:}

If a Para-Sasakian manifold is an $\eta$-Einstein manifold admits a concircular vector field $\xi^{\alpha}$ characterised by the equation (4.1) then the scalars $\tau_{1}$ and $\tau_{2}$ are connected by the relations $\tau_{1}=1+\frac{R}{(n-1)}$ and $\tau_{2}=-\left[n+\frac{R}{(n-1)}\right]$.

Proof:

Transvecting equation (4.1) with $g^{\alpha \beta}$ and on making use of the equation (1.6), consequently, the equation (4.1) assumes the form

(4.2) $n \tau_{1}+\tau_{2}=R$,

Again transvecting equation (4.1) with $g^{\beta \gamma}$ and using the equation (1.5), we get

(4.3) $\tau_{1} \delta_{\alpha}^{\gamma}+\tau_{2} \eta_{\alpha} \xi^{\gamma}=R_{\alpha}^{\gamma}$,

Transvecting equation (4.3) with $\eta_{\gamma}$ and on making use of the equations (1.4) and (1.15), we obtain

(4.4) $\tau_{1}+\tau_{2}=-(n-1)$,

Subtracting equations (4.2) and (4.4), we get

(4.5) $\tau_{1}=1+\frac{R}{(n-1)}$

Inserting the values of $\tau_{1}$ in equation (4.4), we obtain

(4.6) $\tau_{2}=-\left[n+\frac{R}{(n-1)}\right]$.

\section{Definition 4.2:}

A manifold $(\mathrm{M}, \eta, \xi, \mathrm{g})$ is said to be D-homothetic deformation of a Para-Sasakian manifold if the relation

(4.7) $\eta^{*}=a_{1} \eta$,

(4.8) $\xi^{*}=\frac{1}{a_{1}} \eta$

and

(4.9) $g^{*}=a_{1} g+a_{1}\left(a_{1}-1\right) \eta \oplus \eta$

holds good for a positive constant $a_{1}$.

D-homothetic deformation of an $\eta$-Einstein Para-Sasakian manifold satisfying the relation

(4.10) $R_{\alpha a b c} R_{\beta}^{a b c}=\tau_{3} g_{\alpha \beta}+\tau_{4} \eta_{\alpha} \eta_{\beta}$.

Wherein $\tau_{3}$ and $\tau_{4}$ are scalar fields and are given by

(4.11) $\tau_{3}=\left\{(2 n+3) c^{2}+6 n-10\right\}$

and

(4.12) $\tau_{4}=-\frac{1}{2}(n+1)\left(c^{2}-1\right)$.

Wherein $\mathrm{c}=\frac{4 R-(n-1)(3 n-1)}{n^{2}-1}$.

Definition 4.3:

If an $\eta$-Einstein Para-Sasakian manifold (M, $\eta, \xi, \mathrm{g})$ satisfies the relation

(4.13) $R_{\alpha a b c} R_{\beta}^{a b c}=h g_{\alpha \beta}$,

then manifold is said to be constant curvature. 


\section{Definition 4.4:}

Structure $(\mathrm{M}, \eta, \xi, \mathrm{g})$ is said to be Para-Sasakian of constant $\phi$-holomorphic sectional curvature if its tensor field vanishes identically.

\section{Definition 4.5:}

If an $\eta$-Einstein Para-Sasakian manifold $\left(M^{*}, \eta^{*}, \xi^{*}, g^{*}\right)$ satisfying the relation

(4.14) $R_{\alpha a b c}^{*} R_{\beta}^{* a b c}=h^{*} g_{\alpha \beta}$,

is termed as constant $\phi$-holomorphic sectional curvature.

Deformed structure is also a Para-Sasakian structure and its Ricci tensor $R_{\alpha \beta}^{*}$ is given by

(4.15) $R_{\alpha \beta}^{*}=R_{\alpha \beta}-2\left(a_{1}-1\right) g_{\alpha \beta}+\left\{(n-1) a_{1}^{2}+2 a_{1}-n-1\right\} \eta_{\alpha} \eta_{\beta}$

In view of above discussions, the deformed manifold $\left(M^{*}, \eta^{*}, \xi^{*}, g^{*}\right)$ satisfies the following relations

(4.16) $R_{\alpha \beta}^{*}=\tau_{3}^{*} g_{\alpha \beta}$,

and

(4.17) $R_{\alpha a b c}^{*} R_{\beta}^{* a b c}=\tau_{4}^{*} g_{\alpha \beta}$.

Wherein $\tau_{3}^{*}$ and $\tau_{4}^{*}$ are scalar fields.

In this regard, we have the following theorems:

\section{Theorem 4.2:}

Let $\mathrm{M}$ be a D-homothetic deformation of $\eta$-Einstein Para-Sasakian manifold with constant curvature then the curvature tensor satisfies the relation $R_{\alpha a b c} R_{\beta}^{a b c}=\frac{1}{2 n}\left[\left(4 n^{2}+5 n-1\right) c^{2}+\left(12 n^{2}-19 n+1\right)\right] g_{\alpha \beta}$.

\section{Proof:}

If $\mathrm{D}$-homothetic deformation of $\eta$-Einstein Para-Sasakian manifold is constant curvature then from equations (4.10) and (4.13), we obtain

(4.18) $\tau_{3} g_{\alpha \beta}+\tau_{4} \eta_{\alpha} \eta_{\beta}=h g_{\alpha \beta}$,

Contracting equation (4.18) by $g^{\alpha \beta}$ and using equations (1.4) and (1.5) yields

(4.19) $n \tau_{3}+\tau_{4}=n h$,

From equations (4.11), (4.12) and (4.19), we get

(4.20) $h=\frac{1}{2 n}\left[\left(4 n^{2}+5 n-1\right) c^{2}+\left(12 n^{2}-19 n+1\right)\right]$,

By virtue of equations (4.13) and (4.20), we obtain

(4.21) $R_{\alpha a b c} R_{\beta}^{a b c}=\frac{1}{2 n}\left[\left(4 n^{2}+5 n-1\right) c^{2}+\left(12 n^{2}-19 n+1\right)\right] g_{\alpha \beta}$.

\section{Theorem 4.3:}

If $\mathrm{D}$-homothetic deformation of $\eta$-Einstein Para-Sasakian manifold is constant $\phi$-holomorphic sectional curvature then the scalar fields $\tau_{3}^{*}$ and $\tau_{4}^{*}$ are connected by the relations $\tau_{3}^{*}=\frac{1}{n}\left[R-(n-1)\left(a_{1}-1\right)^{2}\right]$ and $\tau_{4}^{*}=h^{*}$.

Proof:

In view of equations (4.15) and (4.16) we observe that

(4.22) $\tau_{3}^{*} g_{\alpha \beta}=R_{\alpha \beta}-2\left(a_{1}-1\right) g_{\alpha \beta}+\left\{(n-1) a_{1}^{2}+2 a_{1}-n-1\right\} \eta_{\alpha} \eta_{\beta}$,

Contracting equation (4.22) by $g^{\alpha \beta}$ and using equation (1.6), we obtain

(4.23) $\tau_{3}^{*}=\frac{1}{n}\left[R-(n-1)\left(a_{1}-1\right)^{2}\right]$,

From equations (4.14) and (4.17), we get

(4.24) $\tau_{4}^{*}=h^{*}$.

\section{References}

1) De K. D-Homothetic deformation of LP-Sasakian manifolds. Novi Sad Journal of Mathematics. 2015;45(2):113-123. doi:10.30755/nsjom.2014.033.

2) Dimri RC, Chauhan TS, Chauhan IS, Srivastava VK. A note on Einstein manifold in P-Sasakian manifold. International transactions in applied sciences. 2010;2(1):161-164.

3) Shaikh AA, Baishya KK, Eyasmin S. On D-Homothetic deformation of trans-Sasakian structure. Demonstratio Mathematica. 2008;XLI(1):171-188.

4) Chauhan I, Chauhan TSS, Singh R, Rizwan MK. A note on D-Conformal para killing vector field in a P-Sasakian manifold. Journal of Xidian University. 2020;14:326-329.

5) Chauhan TS, Chauhan IS, Kumar. Rajendra: On recurrent and birecurrent Para-Sasakian manifolds. Acta Ciencia Indica. 2003;(1):145-148.

6) Matsumoto M. On Riemannian spaces with recurrent projective curvature;vol. 19. Tensor, S N, editors. 1968. 